\title{
Effects of Ticlopidine on Platelet Aggregation in Beagles with Unstable Angina
}

\author{
Weiting Wang*, Chunhua Hao, Zhuanyou Zhao \\ State Key Laboratory of Pharmacokinetics and Pharmacodynamics, Tianjin Institute of Pharmaceutical Research, Tianjin, China \\ Email address: \\ 23006858@163.com (Weiting Wang) \\ ${ }^{*}$ Corresponding author \\ To cite this article: \\ Weiting Wang, Chunhua Hao, Zhuanyou Zhao. Effects of Ticlopidine on Platelet Aggregation in Beagles with Unstable Angina. Science \\ Journal of Clinical Medicine. Vol. 6, No. 1, 2017, pp. 20-23. doi: 10.11648/j.sjcm.20170601.12
}

Received: February 12, 2017; Accepted: March 4, 2017; Published: March 21, 2017

\begin{abstract}
Aim To study the antiplatelet aggregation of ticlopidine (TC). Methods Using coronary artery Folts' model in beagles, the efficacy of TC on platelet aggregation was studied. Results (1)TC16, $32 \mathrm{mg} \cdot \mathrm{kg}^{-1}$ dramatically inhibited the frequency of CFRs dose-dependently, and the times of CFRs decreased by $27 \%, 56 \%, 95 \%, 100 \%$ respectively at $1-4 \mathrm{~h}$ than that in control group's after TC $32 \mathrm{mg} \cdot \mathrm{kg}^{-1}$ administration. CFRs were abolished and did not return when AD was infused after TC $32 \mathrm{mg} \cdot \mathrm{kg}^{-1}$ administration. (2)The time for CFRs absolute disappearance of TC in dose of $32 \mathrm{mg} \cdot \mathrm{kg}^{-1}$ was shorter than that in control group. (3)The platelet aggregation induced by ADP was inhibited obviously after TC $16,32 \mathrm{mg} \cdot \mathrm{kg}^{-1}$ administration, and the aggregation in TC $32 \mathrm{mg} \cdot \mathrm{kg}^{-1}$ didn't return after AD administration. Conclusion TC had dose-dependent antiplatelet effects in beagles, suggesting TC has reliable therapeutical efficacy on angina pectoris.
\end{abstract}

Keywords: Ticlopidine, Antiplatelet Aggregation, Unstable Angina Pectoris

\section{Introdution}

More and more evidence demonstrated that platelet plays an important role in atherosclerosis, and arterial thrombus formation. Antiplatelet treatment became one of the most important techniques for cardio- and cerebro- vascular disease. Unlike aspirin (ASA), Ticlopidinee (TC) was an inhibitor of platelet aggregation acted on some agonists such as ADP, adrenalin (AD), arachidonic acid (AA), thrombin (Th), collagen (CG), 5-HT et al, especifically inhibited ADP-induced platelet aggregation without affecting prostaglandin metabolism. It was extensively applied in unstable angina pectoris (UAP), acute myocardial infarction (AMI), transient ischemic attack (TIA), stroke in clinics $[1,2]$, but still no experimental studies showed that it was effective in models of UAP. So by using Folts' model in beagles, we studied the antiplatelet efficacy of TC.

\section{Materials and Methods}

\subsection{Reagents and Drugs}

TC and ASA were provided by Tianjin Institute of
Pharmaceutical Research, ADP were purchased from Sigma (USA).

\subsection{Animals}

Beagles, weight 7-12 kg, male and female were used, provided by Sin'gang Laboratory Animal Farm (Shanghai, China).

\subsection{Instruments}

RM6300 polygraph physiological recorder, MFV-3200 electromagnetic blood flowmeter, were manufactured by Nihon Kohden Corporation（Tokyo, Japan）, MP100A-CE polychannel biological signal collection system, provided by BIOPAC Systems Inc (California, USA). SC-3 electrical respirator, Shanghai Medical Equipment Factory, SPA- III automatic equilibrium platelet aggregometer, made by Keda Test Instrument Factory (Shanghai).

\subsection{Methods}

\subsubsection{Surgical Preparation}

The surgical preparation has been described in detail in previous publications $[3,4,5]$. Beagles of either sex were 
anaesthetized with sodium pentobarbital $30 \mathrm{mg} \bullet \mathrm{kg}^{-1}$ by intravenous injection, supplemental barbiturate anesthesia (3 $\mathrm{mg} \bullet \mathrm{kg}^{-1}$ ) was administered at 60 min intervals to maintain anaesthesia state. Respiration was supported mechanically via trachea, the chest opened at the left fourth intercostals space, made a hammock to hold the heart, and the left circumflex coronary artery was dissected out 2-3 cm long approximately, and an electromagnetic flow probe (dimension 2-3 $\mathrm{mm}$ ) was placed around the artery conjuncted electromagnetic MFV-3200 flowmeter to measure coronary blood flow. Mean artery blood pressure (MABP) was measured via a polyethylene cannula inserted into right femoral artery and connected via a normal saline -filled tube to a TP-400T pressure transducer. Venous access was via the right femoral vein, with a $0.9 \%$ saline drip administered continuously to maintain blood volume. ECG (lead-II) was measured continuously.

Analogue flow, pressure and ECG signals were amplified via RM6300 polygraph physiological recorder and fed into a MP100A-CE polychannel biological signal collection system for analogue-to-digital converting with collecting frequency 300 HZ. The digital output was then displayed, recorded and processed on a computer running Acknownowledge version 7.2 software (BIOPAC Systems Inc.).

\subsubsection{Stenosis}

When the status remained stable up to $30 \mathrm{~min}$ after surgical preparations, cycle flow reductions (CFRs) were made using the coronary artery Folts' models in dogs. Distal to the probe, the artery was occluded by holding a silk for 20 second, there is a sudden restoration of blood flow when the artery was loosen again, that is "hyperaemic phenomenon of reaction (HPR)", when the HPR disappeared, about 4mm long artery was clamped two times and 5 seconds for one time with a special surgical clamp to produce intimal and medial damage. A plastic cylinder of remodulation in inside diameter was placed around the damaged artery. The HPR was no happened when to produce a $70 \pm 5 \%$ reductions in arterial diameter [6], platelet aggregation periodically in the area of the stenosed and damaged artery lumen gradually forming an occlusive platelet thrombus that causes coronary blood flow to decline to zero. A pressure gradient then builds up across the stenosed lumen, dislodging the platelet thrombus and causing fragment and embolize distal to the stenosed site, and this cause a sudden restoration of blood flow also. This acute platelet mediated thrombus formation followed embolization causes the phenomenon of cyclic flow reduction (CFRs), the frequency of CFRs is a direct indication of in vivo platelet activity [7]. If the thrombus dose not embolize spontaneously, the plastic cylinder is shaken gently to dislodge the thrombus, in order to prevent animal's death with serious myocardial infarction.

\subsubsection{Experimental Protocol}

After the CFRs started, we observed the first 1 hour as control period, the drug was administered via duodenum (the volume was $15 \mathrm{ml}$ ) following to observe next 4 hours, then AD $4 \mu \mathrm{g} \cdot \mathrm{kg}^{-1}$ was given via intravenous infusion for 20 minute (the volume was $20 \mathrm{ml}$ ) [8].

Twenty-four (24) beagles with successful model weighing between 7-12 kg were used for the study and randomly assigned into four groups of six animals in each group. Group1 (control group) was given the distilled water in volume of $15 \mathrm{ml}$, Groups 2 and 3 received orally the TC at doses of $16,32 \mathrm{mg} \cdot \mathrm{kg}^{-1}$ respectively; Group4 (positive control) was given ASA in $32 \mathrm{mg} \cdot \mathrm{kg}^{-1}$. Blood was obtained by cardiac puncture into a sterilized sample bottles for testing ex vivo platelet aggregation.

\subsubsection{Criterion for CFRs, and Time for CFRs Disappearance}

When the coronary blood flow declined to 30 percent of baseline, CFRs happened. We made $95 \%$ confidence of the frequency of control group's CFRs as reference, if the times of drugs' CFRs were lower than that of reference's, the drug showed efficiency. The zero time of CFRs was made as criterion for absolute inhibition, and the time from drug administration to the last appearance of CFRs was made as the time for CFRs disappearance.

\subsubsection{Ex Vivo Platelet Aggregation Studies}

At predeterminated time, $4.5 \mathrm{~mL}$ of whole blood were drawn into a syringe containing $0.5 \mathrm{~mL}$ of $3.8 \%$ sodium citrate as an anticoagulant, blood was centrifuged at 150 $\mathrm{g} \cdot \mathrm{min}^{-1}$ for $10 \mathrm{~min}$ at room temperature in order to get platelet-rich plasma (PRP), the remains were further centrifuged at $1000 \mathrm{~g} \bullet \mathrm{min}^{-1}$ for $10 \mathrm{~min}$ at room temperature to obtain platelet -poor plasma (PPP). The platelet counts of PRP were adjusted to 300,000 cells per microliter by dilution with PPP. PRP 200 micriliters preincubated for $10 \mathrm{~min}$ at $37^{\circ} \mathrm{C}$ were added into colorimetry pool, and tracing pen was adjusted to $0 \%$ with PRP, $100 \%$ with PPP, the platelet aggregation was studied using $\mathrm{ADP}$ (final concentration, 2 $\left.\mathrm{mmol} \cdot \mathrm{L}^{-1}\right)$.

\subsubsection{Statistical Methods}

Data are presented as mean $\pm \mathrm{SD}$ and were analyzed using one-way analysis of variance (ANOVA) followed with a post-hoc test (least significance difference). Enumeration data was expressed as percentage, analysed by Chi-square Fisher's exact test.

\section{Results}

\subsection{Effect of TC on CFRs}

The frequency of CFRs in control group remained baseline in control period (averaged 10.5 $\pm 2.1 / 1 \mathrm{~h}$ ) and $4 \mathrm{hrs}$ after distilled water administration. TC $16,32 \mathrm{mg} \bullet \mathrm{kg}^{-1}$ dramatically inhibited the frequency of CFRs dose-dependently, and the frequency of CFRs decreased by $87 \%, 100 \%$ respectively at 1-4 $\mathrm{h}$ than that in control group's. CFRs were abolished and did not return when AD was infused after TC $32 \mathrm{mg} \cdot \mathrm{kg}^{-1}$ administration (Table 1). The time for CFRs absolute disappearance of TC in dose of $32 \mathrm{mg} \bullet \mathrm{kg}^{-1}$ was shorter than that in control group (Table 2). 
Table 1. The effect of TC on CFRs.

\begin{tabular}{|c|c|c|c|c|c|c|c|}
\hline \multirow{2}{*}{ Group } & \multirow{2}{*}{ Dose $\left(\mathbf{m g} \cdot \mathbf{k g}^{-1}\right)$} & \multirow{2}{*}{ Control period } & \multicolumn{4}{|c|}{ After drug administration $\left(\right.$ times $\left.\cdot h^{-1}\right)$} & \multirow{2}{*}{ AD } \\
\hline & & & 1 & 2 & 3 & 4(hr) & \\
\hline Group1 & - & $10.5 \pm 2.1$ & $11.0 \pm 2.5$ & $10.7 \pm 2.2$ & $9.7 \pm 2.0$ & $9.5 \pm 1.5$ & $11.8 \pm 2.9$ \\
\hline Group2 & TC 16 & $9.8 \pm 1.8$ & $8.7 \pm 1.9$ & $4.8 \pm 1.3^{* * *}$ & $2.0 \pm 1.8^{* * *}$ & $1.2 \pm 1.9^{* * *}$ & $4.5 \pm 1.9^{* * *}$ \\
\hline Group3 & TC 32 & $10.0 \pm 1.5$ & $8.0 \pm 1.8^{*}$ & $4.7 \pm 0.8^{* * *}$ & $0.5 \pm 0.8^{* * *}$ & $0 \pm 0^{* * *}$ & $0.3 \pm 0.5^{* * *}$ \\
\hline Group4 & ASA32 & $9.7 \pm 1.6$ & $7.2 \pm 1.7^{* *}$ & $4.5 \pm 2.4^{* * *}$ & $7.0 \pm 3.5$ & $9.5 \pm 2.1$ & $9.2 \pm 3.1$ \\
\hline
\end{tabular}

$\overline{\boldsymbol{x}} \pm \mathrm{s}, \mathrm{n}=6,{ }^{*} \mathrm{P}<0.05,{ }^{* *} \mathrm{P}<0.01,{ }^{* * *} \mathrm{P}<0.001$ vs control group.

Table 2. The effect of TC on the time for CFRs disappearance.

\begin{tabular}{llll}
\hline Group & Dose $\left(\mathbf{m g}^{*} \mathbf{k g}^{-1}\right)$ & percentage of CFRs disappearance (\%) & Time for CFRs disappearance (min) \\
\hline Group1 & - & 0 & $>240$ \\
Group2 & TC 16 & 50 & $184.8 \pm 63.2$ \\
Group3 & TC 32 & $100^{*}$ & $101.2 \pm 24.2^{* *}$ \\
Group4 & ASA32 & 33.3 & $188.5 \pm 81.8$ \\
\hline
\end{tabular}

$\overline{\boldsymbol{x}} \pm \mathrm{s}, \mathrm{n}=6,{ }^{*} \mathrm{P}<0.05,{ }^{* *} \mathrm{P}<0.01$ vs control group.

\subsection{Effect of TC on Ex Vivo Platelet Aggregation}

The platelet aggregation induced by ADP showed no change in control period and $4 \mathrm{hrs}$ after distilled water administration in control group. The platelet aggregation was inhibited obviously after TC16, $32 \mathrm{mg} \cdot \mathrm{kg}^{-1}$ administration, TC $32 \mathrm{mg} \cdot \mathrm{kg}^{-1}$ could inhibit platelet aggregation about to $57.1 \%$ maximally. The aggregation in TC $32 \mathrm{mg} \cdot \mathrm{kg}^{-1}$ didn't return after $\mathrm{AD}$ administration (Table 3 ).

Table 3. The effect of TC on platelet aggregation (\% of full scale) induced by ADP.

\begin{tabular}{|c|c|c|c|c|c|c|c|}
\hline \multirow{2}{*}{ Group } & \multirow{2}{*}{ Dose $\left(\mathrm{mg} \mathbf{k g}^{-1}\right)$} & \multirow{2}{*}{ Control period } & \multicolumn{4}{|c|}{ After drug administration } & \multirow[t]{2}{*}{ AD } \\
\hline & & & 1 & 2 & 3 & 4(hr) & \\
\hline Group1 & - & $62.2 \pm 7.6$ & $56.8 \pm 4.4$ & $59.2 \pm 5.8$ & $57.0 \pm 5.8$ & $55.2 \pm 7.1$ & $63.0 \pm 10.4$ \\
\hline Group2 & TC 16 & $64.3 \pm 8.8$ & $58.3 \pm 8.4$ & $41.8 \pm 9.9 * *$ & $31.5 \pm 7.5^{* *}$ & $28.2 \pm 8.8 * *$ & $50.8 \pm 11.1 *$ \\
\hline Group3 & TC 32 & $62.8 \pm 9.0$ & $52.5 \pm 7.7$ & $32.7 \pm 5.6^{* *}$ & $22.5 \pm 4.4 * *$ & $23.7 \pm 5.4 * *$ & $21.7 \pm 4.3^{* *}$ \\
\hline Group4 & ASA32 & $63.8 \pm 5.9$ & $50.8 \pm 5.9$ & $49.3 \pm 5.0 *$ & $58.0 \pm 4.8$ & $62.8 \pm 9.6$ & $64.8 \pm 6.3$ \\
\hline
\end{tabular}

$\overline{\boldsymbol{x}} \pm \mathrm{s}, \mathrm{n}=6,{ }^{*} \mathrm{P}<0.05,{ }^{* *} \mathrm{P}<0.01$ vs control group.

\section{Discussion}

The unstable angina pectoris was mostly related to cracking of atherosclerosis clotty [9], and platelet aggregation and releasing were important in coronary arterial thrombus formation [10].

Regardless the thrombus in heart or in artery and veins, its formation process was all been from the platelets adhesion to exposed CG lying on subendothelium surface of injured vessels. When endothelium cell was damaged, the $\mathrm{CG}$, Vwf exposed, which led platelet be adhesive, aggregate, and release (such as ADP, AD, 5-HT, AA, Th, PF et al). The activated platelet not only related to coagulation cascade process, but also released vascular activator, cellular factor, and growth factor, which associated with pathological conditions of unstable angina pectoris, acute myocardial infarction, transient ischemic attack, stroke and play role on acute or chronic thrombus reocclusion and restenosis after interventional therapy [11].

This paper adopted the Folts' model according to this principle, when the lesion coronary artery arrived to critical stenosis for a while, the platelet was to be adhesive, aggregate, release, and the cascade of thrombus and thrombin formation which turn fibrinogen into fibrin was started. The occlusive platelet thrombus caused coronary blood flow to decline to zero. The platelet thrombus was dislodged and dissolved with pressure gradient building up across the stenosed lumen. The CFRs observed in the dog were thought to be correlated well with acute ischemic syndromes in humans [12].

Twinty seven beagles were used for Folts' model in this research, but 3 beagles did not show CFRs phenomenon, which perhaps was related to lowly platelets count in blood, further more, the times of CFRs affected strongly by animal's body temperature. With strict conditions, the frequency of CFRs in this research averaged $10.0 \pm 1.7(n=24)$, and remained stability in whole experiment.

As antiplatelet agent, aspirin showed some disadvantages such as allergic reaction, nausea, vomiting, hallucinations, irritation of stomach and intestines, the harmful to liver and kidney, bleed, inhibiting synthesizes of the PGI2, et al, therefore those disadvantages urged people to look for the more superior treatment agents.

TC together with clopidogrel was one sort of thienopyridine, which could inhibit the platelet aggregation induced by a lot of agonists, especially on aggregation induced by ADP, and its action was independent of PGI2 synthesis [13]. TC inhibited the the first phase of platelet aggregation which was different from other agonists. The research in this paper demonstrated that TC had obvious inhibition of CRFs in canine models, and inhibited platelet aggregation induced by ADP, those data supplied that TC possess a strong potent antiplatlet activity.

The mechanism of TC on inhibition of platelet aggregation 
was unknown for a long time. Up to day, We known two subtypes of ADP receptor lying on surface of platelet, one type had something to do with platelets transmogrification and $\mathrm{Ca}^{2+}$ channel, the other was related to protein $\mathrm{G}$ complex, which decreased the level of cAMP by influenced the signals transmitting in cells, cAMP promoted the $\mathrm{Ca}^{2+}$ in compact piping system to flow into the plasma, and made the GPIIb-IIIa complex receptor uncovered and linked to fibrinogen which prompted the aggregation among platelets. TC mainly exert it's action by the second type of ADP receptor $[14,15]$, though TC was not a antagonist to ADP, or inhibited the syntheses of ADP receptor, but TC perhaps disturbed the function of receptor. Otherwise, TC could inhibit the using of fibrinogen receptors, and decrease the level of cAMP induced by prostaglandin E1 [16].

\section{Conclusion}

In this study, we established angina model in beagles using Folts methods, and the efficacy of ticlopidine on platelet aggregation was studied. TC had dose-dependent antiplatelet effects in beagles, suggesting TC has reliable therapeutical efficacy on angina pectoris.

\section{References}

[1] Schnorbus B, Daiber A, Jurk K, Warnke S, König J, Krahn U, et al. Effects of clopidogrel, prasugrel and ticagrelor on endothelial function, inflammatory and oxidative stress parameters and platelet function in patients undergoing coronary artery stenting for an acute coronary syndrome. A randomised, prospective, controlled study. BMJ Open. 2014 May 6; 4 (5): e005268. doi: 10.1136/bmjopen-2014-005268.

[2] Dasbiswas A, Rao MS, Babu PR, Vijayvergiya R, Nayak R, Dani S, et al. A comparative evaluation of prasugrel and clopidogrel in patients with acute coronary syndrome undergoing percutaneous coronary intervention. J Assoc Physicians India. 2013, 61 (2): 114-116, 126.

[3] Folts JD, Stamler J, Loscalzo J. Intravenous nitroglycerin infusion inhibits cyclic blood flow responses caused by periodic platelet thrombus formation in stenosed canine coronary arteries. Circulation 1991; 83 (6): 2122-7.

[4] Wang Wei-Ting, Zhao Zhuan-You, Liu Hou-Xiao. The Establishment of the Model of Unstable Angina Pectoris and the Evaluation of Heparin Sodium. Journal of China Pharmaceutical University 2002; 33 (supl): 172-175.
[5] Osman HE. Grape juice but not orange or grapefruit juice inhibits platelet activity in dogs and monkeys (macaca fasciularis). J Nutr 1998, 128: 2307-2312.

[6] Maalej, N. Hoden, JE. Folts, JD. Effect of Shear Stress on Acute Platelet Thrombus Formation in Canine Stenosed Carotid Arteries: An In Vivo Quantitative Study. J Throm Thrombolysis 1998, 5 (3): 231-238.

[7] Folts JD, Rowe GG. Epinephrine potentiation of in vivo stimuli reverses aspirin inhibition of platelet thrombus formation in stenosed canine coronary arteries. 1988; 50 (4): 507-16.

[8] Davi, G. and Patrono, C. Platelet Activation and Atherothrombosis. New England Journal of Medicine, 2007, 357 (24), 2482-2494

[9] Becker R C. Platelet Biology: The Role of Platelets in Hemostasis, Thrombosis and Inflammation [J]. American Journal of Cardiology, 2014, 83 (9A): 3E-6E.

[10] Shaker A. Mousa; Ram Kapil; Dun-Xu Mu. Intravenous and Oral Antithrombotic Efficacy of the Novel Platelet GPIIb/IIIa Antagonist Roxifiban (DMP754) and Its Free Acid Form, XV459 Arteriosclerosis, Thrombosis, and Vascular Biology 1999; 19: 2535-2541.

[11] Ikeda H, Koga Y, Kuwano K, Nakayama H, Ueno T, Yoshida N, et al. Cyclic flow variations in conscious dog model of coronary artery stenosis and endothelial injury correlate with acute ischemic heart disease syndromes in humans. J Am Coll Cardiol 1993; 21 (4): 1008-1017.

[12] Zhang S, Sun C, Hu H, He Y, Yao Y, Cao Y, Zeng Z. Effects of Paclitaxel on the Ability of Aspirin and Clopidogrel to Inhibit Platelet Aggregation. Clin Appl Thromb Hemost. 2016, 22 (7): 673-678.

[13] Christ G, Hafner T, Siller-Matula J M, et al. Platelet Inhibition by Abciximab Bolus-Only Administration and Oral ADP Receptor Antagonist Loading in Acute Coronary Syndrome Patients: The Blocking and Bridging Strategy. Thrombosis research, 2013, 132 (1): e36.

[14] Weisman G A, Woods L T, Erb L, et al. P2Y receptors in the mammalian nervous system: pharmacology, ligands and therapeutic potential.[J]. CNS \& neurological disorders drug targets, 2012, 11 (6): 722-38.

[15] Locker G J, Staudinger T, Knapp S, et al. Prostaglandin E1 inhibits platelet decrease after massive blood transfusions during major surgery: influence on coagulation cascade? 1997, 42 (3): $525-531$.

[16] Di Minno G, Cerbone AM, Mattioli PL, Turco S, Iovine C, Mancini M. Functionally thrombasthenic state in normal platelets following the administration of ticlopidine.J Clin Invest 1985; 75 (2): 328-338. 